\title{
Creando un Puente Cultural entre China y Panamá.
}

\section{Dr. Ramón A. Mon}

Ex Director del Programa del Doctorado en Psicología Clínica, Universidad Católica Santa María La Antigua, Panamá, República de Panamá.

Email: ramon0643@gmail.com

"La memoria puede ser una trampa que, creyéndose reminiscencia, en realidad es premonición. Hay momentos en que confundimos nuestros recuerdos con nuestros deseos..."

Carlos Fuentes

Para establecer un puente cultural entre dos grupos étnicos diferentes tenemos que revisar los "momentos de encuentro" entre ambos grupos y los fenómenos psicosociales que se generan a partir dichos encuentros. Podemos iniciar un análisis del encuentro de la cultura china con la cultura panameña tomando como base los datos, fundamentalmente historiográficos con los que contamos al presente y a partir de ahí definir aquel material de análisis que nos falte por estudiar.

Sin entrar en detalles podemos mencionar dos momentos macros en que Asia ha estado en contacto con América y en particular resaltar aquellos encuentros entre los propiamente chinos y los americanos, específicamente con los habitantes de nuestro país.

\section{Lo que tenemos hasta ahora.}

1. La migración inicial de los pueblos originarios de América que provenían de Asia, mayormente de la región de Siberia. Iniciaron el poblamiento de nuestro continente cruzando el puente de Beringia o estrecho de Bering durante el paleolítico superior (15,000 y 20,000 años). Los estudios se han realizado mediante los hallazgos y restos arqueológicos y en el presente gracias a la presencia viva de distintos grupos amerindios a lo largo del continente mediante análisis genéticos.

2. Sin embargo, la migración china a América y a otros países del sudeste asiático durante la segunda mitad del siglo XIX ha sido la mejor documentada por los historiadores. Específicamente, con respecto a nuestro país, se han escrito muy buenos trabajos recopilando la primera época (fechada a partir de 1854) de la migración china al Istmo de Panamá así como las oleadas migratorias subsiguientes. 
Invest. pens. crit. (ISSN 1812-3864)

Vol. 5, No. 3, septiembre-diciembre 2017

pp. $90-95$

Estos escritos, trabajos y esfuerzos ofrecen un excelente sustento para partir de los mismos e iniciar una exploración la estructura conceptual cultural que se ha ido generando a lo largo de 160 años de presencia china en Panamá.

\section{Mencionaré algunas de las más relevantes}

- Juan Tam:

- “Huellas Chinas en Panamá - 150 años de presencia” (2003)

- Berta Alicia Chen P. :

- “Cómo, cuándo y por qué llegaros los chinos a Panamá” (2010)

- "Presencia China en las Construcciones del Ferrocarril y del Canal de Panamá. (1852 1914) Publicado en 2014.

- Ramón Arturo Mon:

- “La Migración China en Panamá (Un recuento histórico)" (1999) Panamá: Biblioteca de la Nacionalidad. Autoridad del Canal de Panamá.

- "Procesos de Integración de la Comunidad China a la Nación Panameña" en ESTE PAÍS, UN CANAL: ENCUENTRO DE CULTURAS. Panamá: Editado por Ileana Golcher. (1999)

- “Sueños de un Bonsai Truncado" (2010) en MIGRACIÓN, RACISMO Y PODER Editado por Isabel Sanfeliu y Jesús Varona. Colección Imago. Madrid: Biblioteca Nueva.

- "El Mito del Eterno Retorno" - Fenomenología de la inmigración y del inmigrante. (2013) En VIAJE A LA COMPLEJIDAD. N 4 Editado por Nicolás Caparrós y Rafael Cruz Roche. Madrid: Biblioteca Nueva.

- 'La Persistencia de la Memoria: de cómo los nietos de inmigrantes chinos recuerdan a sus abuelos (2013). Conferencia dictada para la Sociedad Psicoanalítica de México preparada para el Harvard Latinoamerican Studies.

\section{- Luis Wong Vega:}

- "Vástagos del Dragón: 26 poetas chino-panameños. Editado por Luis Wong Vega. Panamá: Litho Impresora Chen S. A.

- Sociedad Internacional de Estudios de los Chinos de Ultramar (ISSCO)

- No podemos dejar de mencionar la contribución del Ciclo de Conferencias "Del Sur de China al Sur del Continente Americano" celebrado en 2014 por la Sociedad Internacional de Estudio de los Chinos de Ultramar (ISSCO) con la participación de 60 profesionales de las ciencias sociales de doce países y cuya actividad culminó con el obsequio de la Asociación de Profesionales Chino-panameña de una réplica en pequeña escala del barco 
"Bruja del Mar" (30 de marzo de 1854) hoy en el Museo del Centro de Visitantes del Canal en Miraflores.

\section{Lo que nos falta.}

Debemos partir de la pregunta: ¿Se ha creado un puente cultural? Tenemos como punto de partida los muy serios trabajos de la Dra. Lok Siu * y el mío titulado La Persistencia de la Memoria. Contamos igualmente con otros artículos publicados en revistas y periódicos que aunque pequeños o circunstanciales no carecen de valor. Sin embargo, faltan estudios aún más profundos sobre la filosofía, la psicología y la sociología del pueblo chino afincado en Panamá a lo largo de 160 años.

De acuerdo con Triandis (1992) cultura se definiría como "un conjunto compartido de creencias, actitudes, normas, valores y conducta organizado alrededor de un tema central y que encontramos entre los que hablan una misma lengua, durante un período y en alguna región geográfica".

Como ustedes saben la Comunidad China no es homogénea en su constitución, aunque a simple vista lo parezca, ya que está integrada por diversos grupos lingüísticos que provienen de diferentes regiones de China, un que predominan los ciudadanos chinos de Guangdong (Cantón). Los chinos de Panamá mantienen distintas formas de entender la realidad y hablan diferentes lenguas como el Cantonés y sus derivados, el Hakka y el Mandarín. También influye en la heterogeneidad de la comunidad la generación inmigrante a la que se pertenece es decir, cuándo llegaron y su estatus migratorio. Esta inmigración se ha dado mediante lo que podríamos llamar "oleadas migratorias" y ha correspondido a situaciones histórico-sociales que se han dado tanto en China como en Panamá. Nos referimos a eventos políticos cataclísmicos que se dieron en la China del siglo XIX y XX y que coincidieron con los magnos proyectos de construcción correspondientes en el Istmo de Panamá y que se convirtieron en poderosos imanes para los inmigrantes.

Un ejemplo personal de esta heterogeneidad: pertenezco a una cuarta generación de chinos radicados en Panamá y descendiente de una de las primeras oleadas migratorias, dado que mi bisabuelo llegó en el siglo XIX a trabajar durante la construcción del canal francés; y por lo tanto mis hijos resultan ser ya una quinta generación y mis nietos una sexta. Como podrán suponer los inmigrantes chinos y sus descendientes experimentan el "ser chino" desde una realidad diferente teñida por la memoria del pasado, las experiencias del presente y las expectativas del futuro. Los chinos que viven en nuestro país se plantean, consciente o inconscientemente qué significa ser chino, chino-panameño o panameño de origen chino. Cuándo se les pregunta: ¿qué eres? La respuesta más común es: soy chino o chino-panameño o simplemente panameño. Todas las respuestas anteriores son correctas porque en ocasiones se refieren al origen étnico a que pertenecen o con el cual se identifican y en otras ocasiones se refieren a la nacionalidad y/o la cultura. Y por supuesto, la respuesta parece estar influida por las experiencias y circunstancias histórico-sociales que les haya tocado vivir.

- “El Ferrocarril, la Tienda y el Barrio” en CUANDO ORIENTE LLEGÓ A AMÉRICA. Washington D. C.: Banco Interamericano de Desarrollo, 2004.

- "Memories of a Future Home. Diasporic citizenship of Chinese in Panama. California: Stanford University Press, 2005. 
Invest. pens. crit. (ISSN 1812-3864)

Vol. 5, No. 3, septiembre-diciembre 2017

pp. $90-95$

La complejidad de este encuentro entre dos culturas se complica bastante porque los panameños somos en realidad culturalmente seres complejos. Esto es, geográficamente somos centroamericanos, históricamente suramericanos y culturalmente caribeños. Situación difícil de asumir pero es la complejidad que nos define y tiene tanto valor como cualquiera otra identidad nacional. Y cito a Carlos Fuentes que sostiene que "la verdadera nacionalidad se hace y se mantiene por la cultura". Y como la cultura evoluciona con el tiempo podríamos decir: No somos... estamos siendo"

Esta porosidad del tejido social panameño es producto de la complejidad anteriormente mencionada, lo que ha permitido también que los extranjeros lleguen a nuestras tierras no sientan que se estrellan contra una valla o muralla impenetrable. (Mencionar el caso de los subsaharianos que emigran a Europa que no comparten ni la cultura, ni el idioma, ni la religión ni la etnia).

Considero que la memoria es fundamental en la conformidad de nuestra mismidad definida como lo que fuimos, lo que somos y lo que queremos ser. Esta mismidad está en el núcleo central de la cultura de un pueblo. Como psicoanalista se me presentó el reto de contribuir a este proceso de reflexión de la Comunidad China a pedido de una universidad norteamericana y diseñé un estudio que titulé: "La Persistencia de la Memoria: de cómo los nietos recuerdan a sus abuelos inmigrantes".

Resultó finalmente un estudio que pretendió ser un proto-análisis, de los muchos que se podían hacer sobre la memoria de la emigración o del exilio en su relación con la construcción de la identidad personal. Nos pareció interesante conocer cómo se trasmiten tras-generacionalmente ciertos rasgos de la personalidad por aprendizaje explícito o implícito, de patrones de conducta, creencias y también de prejuicios.

La muestra estudiada estuvo compuesta por nietos de inmigrantes chinos que llegaron a Panamá durante los siglos XIX y XX. Queríamos conocer los hechos y las emociones que persistían en la memoria de estos nietos y que de alguna forma influían, al menos en parte, en la identidad y en el destino de los mismos. El estudio no tomó en cuenta la trasmisión transgeneracional1 en la figura de los padres, inmigrantes de primera y segunda generación en el país, sino en su calidad de puentes y trasmisores de hechos vitales. Somos conscientes de que no le hacemos justicia al rol de los padres en la formación directa de la identidad de sus hijos, pero el estudio quería valorar "la persistencia en la memoria", esto es, la fuerza que despliegan las huellas de memoria desde el pasado y que son trasmitidas desde los abuelos. Abuelos, que en ocasiones asumieron el rol de cuidadores primarios.

Trabajamos grabando los comentarios y recuerdos de los sujetos sin intervenir directamente ni sugerir nada. De las entrevistas obtuvimos diez tópicos Importantes para trabajar:

1 Ver en P. Fonagy "Persistencia transgeneracional del apego: una nueva teoría. 


\section{Cuadro de Tópicos:}

1. Apegos y separaciones tempranas

2. Calidad del vínculo establecido con el abuelo/a

3. Procesos de identificación con dichas figuras

4. El idioma como medio de identificación

5. Sueños. Ansiedades y otras manifestaciones conductuales

6. Importancia de la figura del hombre sobre la mujer y otras actitudes similares

7. Importancia del trabajo, el estudio y la comida

8. La presencia de "otra" familia, anterior o simultánea

9. Actitudes racistas o prejuicios

10. El Desconocimiento de la cultura ambiental

Las reflexiones que podemos hacer a partir del análisis del cuadro de Tópicos Importantes, recoge las vivencias de los sujetos participantes. Está claro que varios de los rasgos de la personalidad de los nietos e hijos de inmigrantes entrevistados pueden encontrarse en general también en otras personas, solo que en el microcosmos de los inmigrantes sobresalen y se hacen más visibles.

Esta arqueología de la memoria que los participantes nos regalaron contiene un material tan rico y prometedor en términos de análisis psicológico que nos retrotrae a las palabras de un amigo, psicoanalista catalán, Rogelio Almengor (2010) cuando dice:

"el ser humano es su memoria. La individualidad y su conciencia se originan en ella, pues si la perdiera dejaría de ser lo que es."

El análisis contiene referencias sobre la Identidad, pensando que la Identidad se nutre de la memoria. Los sujetos (de los cuales poseemos registros muy interesantes pero que rebasan los límites de estas reflexiones) reconocían que muchos de sus rasgos de personalidad provenían de los abuelos; por ejemplo, la fortaleza física, el silencio, el feminismo, las obsesiones y otros rasgos de conducta. Predominaba sin embargo, una identidad de base, la pertenencia a la etnia y cultura china. Debemos también señalar que en los inmigrantes de un grupo étnico minoritario, al ser de un fenotipo diferente al de los miembros de la sociedad receptora mayoritaria, la confusión en la identidad se da más a nivel de lo psicológico-conductual que de lo físico; puesto que las diferencias son evidentes.

\section{Entre los rasgos predominantes encontrados podemos señalar:}

- Presentan dificultades en el establecimiento de los vínculos afectivos y en el desarrollo de relaciones de apego seguros. Este fenómeno crea una reacción de alejamiento o distancia emocional que observamos con frecuencia en el grupo chino.

- Se observa el sacrificio de la vida familiar y afectiva debido al trabajo duro y a los esfuerzos por sobrevivir, lo que exige siempre un mayor esfuerzo y resulta una 
Invest. pens. crit. (ISSN 1812-3864)

Vol. 5, No. 3, septiembre-diciembre 2017

pp. $90-95$

limitación en la interacción social. Las familias, por ejemplo, se visitan los domingos y días feriados en el negocio.

- Los descendientes de inmigrantes también sueñan con personas que han muerto y con relaciones que han perdido, añorando especialmente a los abuelos (y por ende a la China) que fueron las figuras que sustituyeron a los padres en la crianza y en el amor.

- Estas observaciones pueden servir de base para estudiar y comprender rasgos culturales del grupo chino y poderlos contrastar con los aspectos diferentes y similares de la cultura de contraste, es decir, de la cultura panameña.

\section{Bibliografía}

Armengol, R. (2010). Felicidad y Dolor. Una mirada ética. Barcelona, España: Editorial Ariel.

Chen, Berta Alicia (2010) “Cómo, cuándo y por qué llegaros los chinos a Panamá” Panamá: $\mathrm{MDC}$

Chen, Berta Alicia (2014). "Presencia China en las Construcciones del Ferrocarril y del Canal de Panamá. (1852 - 1914). Panamá: MDC

Matsumoto, David (1997) CULTURE AND MODERN LIFE. California: Brooks Cole Publishing Co.

Mon, Ramón Arturo. “La Migración China en Panamá (Un recuento histórico)” (1999) Panamá: Biblioteca de la Nacionalidad. Autoridad del Canal de Panamá.

Mon, R. A. (1999) "Procesos de Integración de la Comunidad China a la Nación Panameña" en ESTE PAÍS, UN CANAL: ENCUENTRO DE CULTURAS. Panamá: Editado por Ileana Golcher.

Mon, R. A. (2010) "Sueños de un Bonsai Truncado" en MIGRACIÓN, RACISMO Y PODER - Editado por Isabel Sanfeliu y Jesús Varona. Colección Imago. Madrid: Biblioteca Nueva.

Mon, R. A. (2013) "El Mito del Eterno Retorno" - Fenomenología de la inmigración y del inmigrante. En VIAJE A LA COMPLEJIDAD. No 4 Editado por Nicolás Caparrós y Rafael Cruz Roche. Madrid: Biblioteca Nueva.

Mon, R. A. (2013). "La Persistencia de la Memoria: de cómo los nietos de inmigrantes chinos recuerdan a sus abuelos. Conferencia dictada para la Sociedad Psicoanalítica de México.

Siu, Lok (2004) “El Ferrocarril, la Tienda y el Barrio" en CUANDO ORIENTE LLEGÓ A AMÉRICA. Washington D. C.: Banco Interamericano de Desarrollo, 2004.

Siu, Lok (2005) "Memories of a Future Home. Diasporic citizenship of Chinese in Panama. California: Stanford University Press,

Tam, Juan (2006) “Huellas Chinas en Panamá - 150 años de presencia”. Panamá: Único Impresores.

Triandis, H. C. (1972) The analysis of subjective culture. New York: Wiley 